\title{
ON A SUBCLASS OF CLOSE-TO-CONVEX HARMONIC MAPPINGS
}

\author{
Manivannan Mathi And Jugal Kishore Prajapat*
}

Abstract. For $\alpha>-1$ and $\beta>0$, let $\mathscr{B}_{\mathscr{H}}^{0}(\alpha, \beta)$ denote the class of sense preserving harmonic mappings $f=h+\bar{g}$ in the open unit disk $\mathbb{D}$ satisfying $\left|z h^{\prime \prime}(z)+\alpha\left(h^{\prime}(z)-1\right)\right| \leqslant \beta-\mid z g^{\prime \prime}(z)+$ $\alpha g^{\prime}(z) \mid$. First, we establish that each function belonging to this class is close-to-convex in the open unit disk if $\beta \in(0,1+\alpha]$. Next, we obtain coefficient bounds, growth estimates and convolution properties. We end the paper with applications and will construct harmonic univalent polynomials belonging to this class.

Mathematics subject classification (2010): 30C45, 30C55, 31A05.

Keywords and phrases: Analytic, univalent, starlike, convex functions, close-to-convex functions, subordination, coefficient estimates, convolution.

\section{REFERENCES}

[1] S. V. Bharanedhar And S. Ponnus Amy, Coefficient conditions for harmonic univalent mappings and hypergeometric mappings, Rocky Mountain J. Math 44 (2014), 753-777.

[2] D. Bshouty, S. S. Joshi AND S. B. Joshi, Radius of close-to-convexity harmonic mappings, Complex Var. Elliptic Equ. 58 (2013), 1195-1199.

[3] J. Clunie And T. Sheil-Small, Harmonic univalent functions, Anna. Acad. Sci. Fenn. Ser. A I Math. 9 (1984), 3-25.

[4] M. DorfF, Convolutions of planar harmonic convex mappings, Complex Var. Theory Appl. 45 (2001), 263-271.

[5] N. Ghosh And A. VASUdeVARAO, Some basic properties of certain subclass of harmonic univalent functions, Complex Var. Elliptic Eqn. 63 (2018), 1687-1703.

[6] S. Yu. Graf, On the Schwarzian norm of harmonic mappings, Probl. Anal. Issues Anal. 5 (23) (2016), 20-32.

[7] R. Herandez And M. J. Martin, Stable geometric properties of analytic and harmonic functions, Math. Proc. Cambridge Philos. Soc. 155 (2013), 343-359.

[8] S. Kanas, S. Maharana AND J. K. Prajapat, Norm of the pre-Schwarzian derivative, Bloch's constant and coefficient bounds in some classes of harmonic mappings, J. Math. Anal. Appl. 474 (2019), 931-943.

[9] R. Kumar, M. DorfF, S. GuPTA, AND S. Singh, Convolution properties of some harmonic mapping in the right half plane, Bull. Malays. Math. Sci. Soc. 39 (2016), 439-455.

[10] H. LEWY, On the non-vanishing of the Jacobian in certain one-to-one mappings, Bull. Amer. Math. Soc. 42 (1983), 689-692.

[11] L. Li And S. Ponnus amy, Note on the convolution of harmonic mappings, Bull. Austral. Math. Soc. 99 (2019), 421-431.

[12] L. Li AND S. PonNuSAmy, Injectivity of sections of univalent harmonic mappings, Nonlinear Analysis 89 (2013), 276-283.

[13] Z. LiU And S. Ponnusamy, Radius of fully starlikeness and fully convexity of harmonic linear differential operator, Bull. Korean Mat. Soc. 55 (2018), 819-835.

[14] Z. LIU AND S. PonNUSAMY, Univalency of convolutions of univalent harmonic right half-plane mappings, Comput. Methods Funct. Theory Soc. 17 (2) (2017), 289-302.

[15] T. H. MacGregor, A class of univalent functions, Proc. Amer. Math Soc. 15 (1964), 311-317.

[16] S. Ponnus amy, Pólya Schoenberg conjecture by Carathéodory functions, J. London Math. Soc. 51(2) (1995), 93-104. 
[17] S. Ponnusamy, Differential subordination concerning starlike functions, Proc. Indian Acad. Sci. (Math. Sci.) 104 (1994), 397-411.

[18] S. Ponnusamy, A. Rasila, And A. Sairam Kaliraj, Harmonic close-to-convex functions and minimal surfaces, Complex Var. Elliptic Equ. 59 (7) (2014), 986-1002.

[19] S. Ponnusamy, J. K. Prajapat, and A. Sairam Kaliraj, Uniformly starlike and uniformly convex harmonic mappings, J. Anal. 23 (2015), 121-129.

[20] S. Ponnusamy AND F. Ronning, Srarlikeness properties for convolutions involving hypergeometric series, Ann Univ Mariae Curie-Sklodowska Sect A. 52 (1998), 141-155.

[21] S. Ponnusamy And V. Singh, Criteria for univalent, starlike and convex functions, Bull. Belg. Math. Soc. Simon Stevin 9 (2002), 511-531.

[22] S. Ponnus amy, H. Yamamoto and H. Yanagihara, Variability regions for certain families of harmonic univalent mappings, Complex Var. Elliptic Equ. 58 (1) (2013), 23-34.

[23] J. K. Prajapat, M. Manivannan and S. Maharana, Harmonic mappings with analytic part convex in one direction, J. Anal. 28 (2020), 961-972.

[24] St. Ruschewey And T. Sheil-Small, Hadamard products of schlicht functions and the PólyaSchoenberg conjecture, Comment. Math. Helv 48 (1973), 119-135.

[25] R. Singh AND S. Singh, Convolution properties of a class of starlike functions, Proc. Amer. Math. Soc. 106 (1989), 145-152.

[26] N. M. Temme, Special Function, An Introduction to the classical Functions of Mathematical Physics, Wiley Publ., New York, 1996. 